\title{
Polarimetric fiber laser sensors
}

\author{
H. K. Kim and S. K. Kim \\ Department of Physics, Korea Advanced Institute of Science and Technology, 373-1, Kusong-Dong, Yusung-Ku, Taejon, Korea
}

\author{
H. G. Park
}

Department of Physics, Chonbuk National University, 560-756, Chonju, Korea

B. Y. Kim

Department of Physics, Korea Advanced Institute of Science and Technology, 373-1, Kusong-Dong, Yusung-Ku, Taejon, Korea

Received July 27, 1992

\begin{abstract}
The beating between the eigenpolarization modes from a fiber laser is used to demonstrate an active, polarimetric sensor with direct frequency output. For the measurement of lateral stress applied to the jacketed Nd-doped fiber laser, a sensitivity of $4.8 \mathrm{kHz} / \mathrm{g}$ with a good linearity is obtained.
\end{abstract}

Rare-earth-doped fibers have been extensively investigated for their applications to optical amplifiers and fiber lasers. The Er-doped fiber amplifiers are about to be implemented in practical communication systems. ${ }^{1}$ On the other hand, current use of fiber lasers is relatively limited, although their potential as light sources for telecommunications and fiber-optic sensors is considered to be significant. One example of fiber laser applications is the amplified spontaneous emission source with broad bandwidth and stable optical spectrum ideal for fiber-optic gyroscopes. ${ }^{2}$ Many other forms of the fiber lasers have also been investigated, such as single-frequency lasers, ${ }^{3}$ mode-locked lasers with femtosecond pulse width, ${ }^{4}$ and wavelength-tunable lasers. $^{5}$ In this Letter we propose and demonstrate a new use of the fiber laser as a fiber sensor based on the beating of two mutually orthogonal eigenpolarization modes in the fiber laser cavity. The change in the birefringence of the laser fiber in response to the external perturbations directly translates into a change in the beat frequency of the two polarization modes. The measurement of the beat frequency can be made by using a conventional frequency counter, which leads to a straightforward electronic signal processing. This approach avoids complicated electronic signal processing required for conventional interferometric sensors, ${ }^{6,7}$ in which the optical phase shift information has to be extracted from the direct intensity output having a sinusoidal dependence on the phase shift.

Although the polarization behavior of a laser with anisotropic cavity can be complicated owing to the gain medium, most of the features of our interest could be explained from the polarization properties of a passive cavity. ${ }^{8}$ Assuming no polarizationselective components and no nonreciprocal components inside the Fabry-Perot-type cavity with mirrors at each end, we can represent the Jones matrix of the cavity for one round trip as $A^{T} A$ in a laboratory frame, where $A$ represents the propagation of light in one direction along the fiber cavity with random birefringence and $A^{T}$ represents the transpose matrix of $A$. As expected, two orthogonal eigenpolarization states with different eigenvalues can be obtained for the cavity matrix $A^{T} A$. In this case the light beams in the eigenpolarization states return to their original polarization states after a complete round trip, and the eigenvalues represent corresponding resonance frequencies. It is interesting to note that the two eigenpolarization states are found to be linearly polarized at the mirror position. Figure 1 shows the longitudinal mode lines for each polarization mode and their beat frequencies, where $c$ is the velocity of light in a vacuum, $L$ is the cavity length, $M$ and $N$ are integers, and $n_{X}$ and $n_{Y}$ are the effective refractive indices of the two ( $X$ and $Y$ ) eigenpolarization modes. In general, the two effective refractive indices for the eigenpolarization modes are different, which results in differences in resonance frequencies and their longitudinal mode spacing. Polarization mode beat (PMB) frequencies appear at the symmetric places $\Delta \nu_{\mathrm{pol}}$ and $\Delta \nu_{0}-\Delta \nu_{\mathrm{pol}}$ around adjacent longitudinal

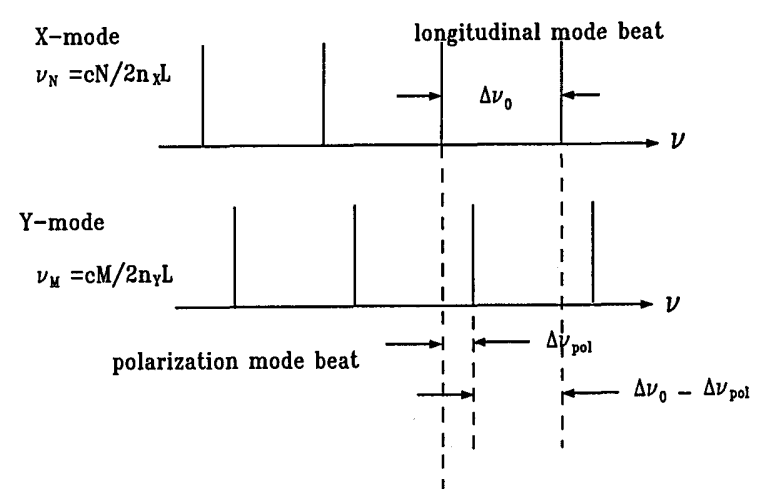

Fig. 1. Resonance frequencies of each eigenpolarization mode and their mode beat frequencies. 


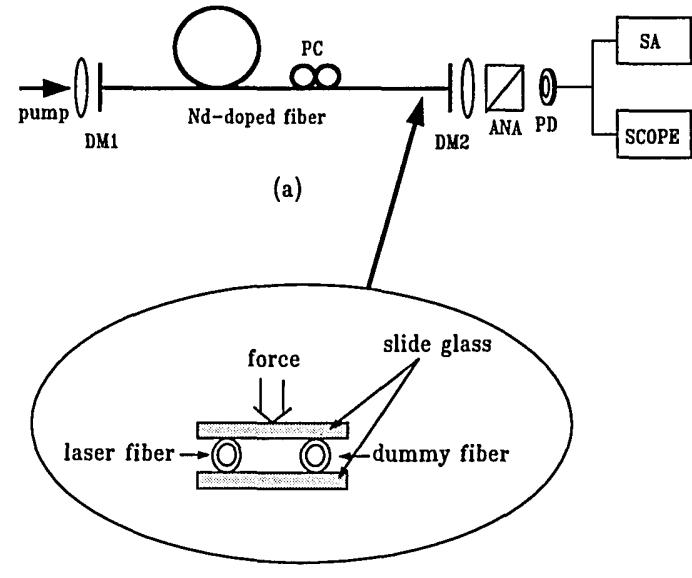

(b)

Fig. 2. (a) Schematic of the experimental setup. DM1, DM2, dichroic mirrors $[R>99 \%$ (DM1) and $R=90 \%$ (DM2) at $1.06 \mu \mathrm{m}$, and $T=80 \%$ at $514.5 \mathrm{~nm}$ ]; PC, polarization controller; ANA, analyzer; $\mathrm{PD}$, silicon photodiode; $\mathrm{SA}$, rf spectrum analyzer; SCOPE, oscilloscope. (b) The cross-sectional view of the stressed region.

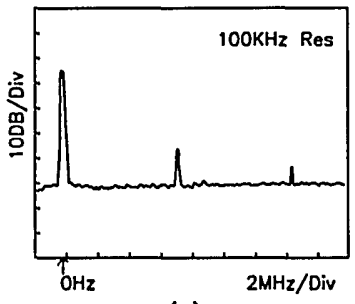

(a)

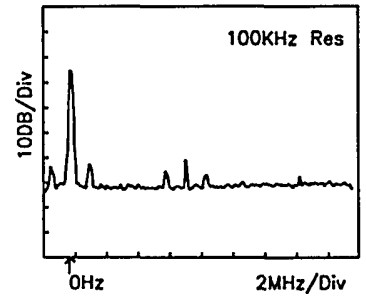

(b)

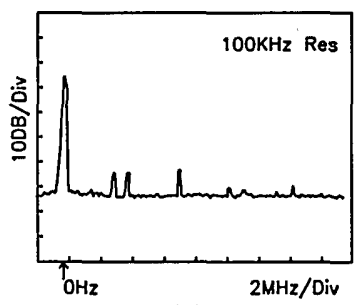

(c)

Fig. 3. Laser output signals monitored through the $\mathrm{rf}$ spectrum analyzer (a) without a polarization analyzer and (b), (c) with a polarization analyzer for different polarization controller settings.

mode beat (LMB) frequencies $\Delta \nu_{0}$. The change in $\Delta \nu_{\mathrm{pol}}$ owing to the change of birefringence in the fiber cavity can be easily monitored by mixing the two polarization states with a polarizer.

Figure 2(a) shows the experimental setup. The Nd-doped circular core fiber (provided by BT Laboratories) had a length of $13 \mathrm{~m}$, a cutoff wavelength of $920 \mathrm{~nm}$, and a Nd concentration of $\sim 200$ parts in $10^{6}$. Dichroic mirrors were glued to the polished ends of the fiber in capillary tubes. The pump source was an $\mathrm{Ar}^{+}$laser at $514.5 \mathrm{~nm}$, and a polarization controller was placed near the output mirror. The pump power used for our experiments was $\sim 1.3$ times the threshold pump power, and the linewidth of the optical spectrum of the fiber laser output was $\sim 10 \mathrm{~nm}$ centered at $1.06 \mu \mathrm{m}$. The laser output signal was detected by a silicon photodiode behind the analyzer, which was followed by a rf spectrum analyzer and an oscilloscope.

Figure 3(a) shows the LMB signal on the rf spectrum analyzer, detected without the analyzer. The mode spacing was measured to be $\sim 7.5 \mathrm{MHz}$, in agreement with $c / 2 n L$. When the analyzer was inserted in front of the detector, a new beat signal appeared at symmetric positions between the LMB signals as seen in Fig. 3(b). Since polarization mode beat frequency depends on the cavity birefringence, it can be changed by adjusting the polarization controller [Fig. 3(c)]. When the analyzer was rotated, the PMB signals disappeared at two particular analyzer angles separated by $90 \mathrm{deg}$, which varied as a function of the setting of the polarization controller. This observation confirms the theoretical prediction that the eigenpolarization states at the output mirror are linearly polarized. In our experiment, pump polarization dependence of the laser output polarization state $^{9}$ was negligible, probably owing to the multimode propagation of the pump beam in the fiber. Polarization mode competition phenomena previously observed in a relaxation oscillation pattern ${ }^{10}$ was also observed in this experiment.

In order to operate the fiber laser as a sensor, we measured the amount of PMB frequency change as a function of the lateral stress applied to the 7.5$\mathrm{cm}$-long section of the jacketed fiber just before the output mirror, as shown in Fig. 2(b). The diameters of the fiber were $126 \mu \mathrm{m}$ (unjacketed) and $250 \mu \mathrm{m}$ (jacketed). One of the eigenpolarization directions at the laser output was adjusted to be parallel to the direction of the lateral stress using the polarization controller. Figure 4 shows the PMB frequency shift versus the applied stress, which was read from the rf spectrum analyzer. As expected, the PMB frequency change was linearly proportional to the lateral stress with a slope coefficient of $4.8 \mathrm{kHz} / \mathrm{g}$. A theoretical estimate could be made by using the stress-optic effect for single-mode fiber ${ }^{11}$ and the relation $\Delta \nu_{\mathrm{pol}}=(c / 2 n L)(\phi / 2 \pi)$, where $\phi$ is the relative phase change between the two eigenpolarization modes due to the lateral stress. For a diameter of $126 \mu \mathrm{m}, \Delta \nu_{\mathrm{pol}}$ should be $11.7 \mathrm{kHz} / \mathrm{g}$, and for a 250 $\mu \mathrm{m}$ diameter, this value becomes $6 \mathrm{kHz} / \mathrm{g}$, both of which are greater than the experimental results.

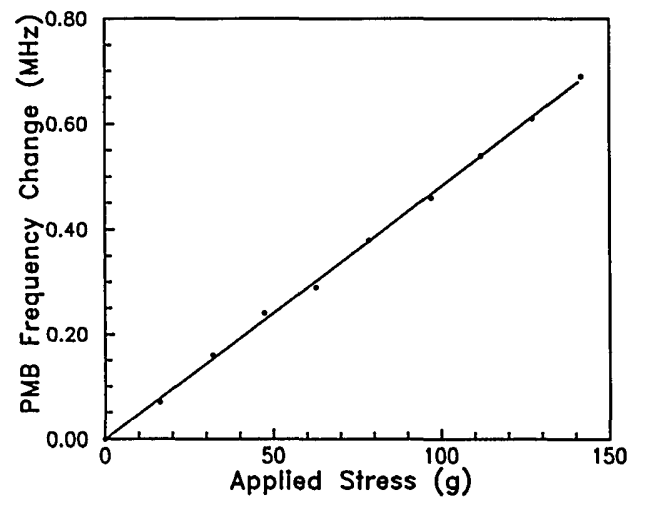

Fig. 4. Shift of PMB frequency versus applied stress. 


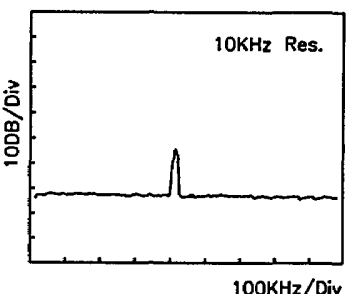

(a)

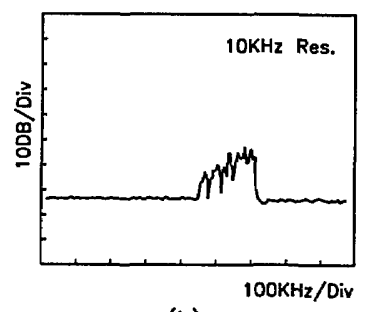

(b)
Fig. 5. Typical bandwidths of (a) LMB signal and (b) PMB signal.

The origin of this difference is probably due to the soft jacket that deforms under the lateral stress.

The bandwidth of PMB signal is an important parameter for the fiber laser sensor, since it determines the sensitivity and accuracy of the sensor. Figures 5(a) and 5(b) show the bandwidths of the LMB signal $\left(\Delta f_{L}\right)$ and the PMB signal $\left(\Delta f_{P}\right)$, respectively. The $\Delta f_{L}$ was $\sim 30 \mathrm{kHz}$, and $\Delta f_{p}$ ranged from a few tens to a few hundred kilohertz, depending on the setting of the polarization controller, the linewidth of the optical spectrum, and the magnitude of the birefringence in the fiber. One of the obvious reasons for the relatively broad $\Delta f_{L}$ and $\Delta f_{P}$ is the wavelength dispersion in the fiber and others such as frequency pulling in the gain medium. The upper bound of $\Delta f_{L}$ from the wavelength dispersion could be estimated as $\Delta f_{L} \simeq(c / 2 n L)\left\{\left[n\left(\lambda_{1}\right)-n\left(\lambda_{2}\right)\right] / n\right\}$, where $\lambda_{1}$ and $\lambda_{2}$ are the wavelengths of the lower and upper limits of the laser output spectrum and $n$ is the average refractive index. If the polarization dispersion between the $X$ and $Y$ modes is greater than the wavelength dispersion, $\Delta f_{L} \approx(c / 2 n L)\left[\left(n_{X}-\right.\right.$ $\left.\left.n_{Y}\right) / n\right]$. Since the maximum birefringence induced by bending was estimated to be $10^{-7}$ in our experimental setup, which is far smaller than the wavelength dispersion, $\Delta f_{L}$ is expected to be determined by wavelength dispersion. Although a small birefringence in the fiber laser cavity does not affect the $\Delta f_{L}$, it can cause a serious broadening of $\Delta f_{P}$ when the optical bandwidth is broad and many longitudinal modes are lasing for each polarization modes. In this case,

$$
\Delta f_{P}=\frac{c}{2 L}\left(\frac{1}{n_{X}}-\frac{1}{n_{Y}}\right)\left(\frac{\delta \nu}{c / 2 n L}\right)=\frac{n_{X}-n_{Y}}{n} \delta \nu,
$$

where $\delta \nu$ is the optical bandwidth of the laser output and $n$ is the average refractive index of the $X$ and $Y$ modes. If only one longitudinal mode is operated for each polarization state, $\Delta f_{P}$ should be similar to $\Delta f_{L}$. As a numerical example, for birefringence $\Delta n \simeq 10^{-7}$ and $\delta \lambda \simeq 10 \mathrm{~nm}$ centered at $1.06 \mu \mathrm{m}$ with $n(1.055 \mu \mathrm{m}) \simeq 1.4497434$ and $n(1.065 \mu \mathrm{m}) \simeq$ 1.449622 for fused silica, $\Delta f_{L} \simeq 700 \mathrm{~Hz}$ and $\Delta f_{P} \simeq$ $500 \mathrm{kHz}$. While the value of $\Delta f_{P}$ is in agreement with the experimental result, the experimental $\Delta f_{L}$ is much greater than the theoretical value when only the wavelength dispersion is considered. The discrepancy for $\Delta f_{L}$ might be from other active phenomena in the gain medium. Ideally the $\Delta f_{P}$ should be sufficiently narrow in order to provide desired sensitivity of the fiber laser sensor, which could be obtained by operating the laser with a small number of longitudinal modes by using a narrowband filter inside the cavity.

In conclusion, we have proposed and demonstrated a new use of fiber lasers as sensors with a frequency output. The promising experimental results and theoretical analysis show that a narrow-band laser is desirable for this purpose. When a polarizationmaintaining fiber is used, physical parameters such as strain and temperature that change the birefringence of the fiber can easily be measured with frequency readout.

We thank BT Laboratories for providing the Nd-doped fiber. This research was supported by the Agency for Defense Development under contract 90-1-1.

\section{References}

1. K. Nakagawa, S. Nishi, K. Aida, and E. Yoneda, IEEE J. Lightwave Technol. 9, 198 (1991).

2. K. A. Fesler, R. F. Kalman, M. J. F. Digonnet, B. Y. Kim, and H. J. Shaw, Proc. Soc. Photo-Opt. Instrum. Eng. 1171, 346 (1989).

3. P. Barnsley, P. Urquhart, C. Miller, and M. Brierley, J. Opt. Soc. Am. A 5, 1339 (1988).

4. D. J. Richardson, R. I. Laming, D. N. Payne, M. W. Phillips, and V. J. Matsas, Electron. Lett. 27, 730 (1991).

5. C. R. Cochlain and R. J. Mears, Electron. Lett. 28, 124 (1992).

6. D. A. Jackson, J. Phys. E 18, 981 (1985).

7. F. Maystre and R. Dändliker, Appl. Opt. 28, 1995 (1989).

8. F. Bretenater and A. L. Floch, Opt. Commun. 79, 314 (1990), and references therein.

9. J. T. Lin, W. A. Gambling, and D. N. Payne, in Digest of Conference on Lasers and Electro-Optics (Optical Society of America, Washington, D.C., 1989), paper TUJ25.

10. J. T. Lin and W. A. Gambling, Proc. Soc. Photo-Opt. Instrum. Eng. 1373, 42 (1990).

11. Y. Namihira, M. Kudo, and Y. Mushiake, Electron. Commun. Jpn. 60-C, 107 (1977). 